\title{
Pluronic lecithin organogel with d-limonene as a transdermal delivery system for Kaempferia parviflora extract
}

\author{
Worranan Rangsimawong, Paisit Wattanasri, Prasert Akkaramongkolporn, Prasopchai Tonglairoum, \\ Tanasait Ngawhirunpat and Praneet Opanasopit ${ }^{*}$ \\ Department of Pharmaceutical Technology, Faculty of Pharmacy, Silpakorn University, Nakhon Pathom 73000, Thailand
}

\begin{abstract}
Kaempferia parviflora (KP) extract has been used in the Thai medicinal plant recipe, which the methoxyflavones are the main active compound. These compounds have low water solubility, high lipophilicity, and low bioavailability. The aim of this study was to develop the pluronic lecithin organogel (PLO) and PLO with d-limonene (PLO-L) for enhancing transdermal delivery of KP extract. These formulations were prepared and their physicochemical properties, stability, and in vitro skin permeation were evaluated. For the result, all formulations exhibited good physicochemical properties and stable under storage condition for 3 months. The permeation of KP extract-loaded PLO-L and PLO formulation showed significantly higher total methoxyflavones permeated through the skin than KP extract in water, which PLO-L provided the highest permeated flux of total methoxyflavones. This result suggested that d-limonene play a role as skin permeation enhancer. Organogel consisting of poloxamer 407 and lecithin also increased the skin permeation of KP extract. In conclusion, PLO-L could be a potential transdermal delivery system for KP extract.
\end{abstract}

\section{Introduction}

Kaempferia parviflora (KP) that is commonly known as "krachaidum", or "black ginger", was previously demonstrated to have a number of physiological effects, including male sexual enhancement, antimicrobial, aphrodisiac effect, antigastric ulcer, antidepressant, anticholinesterase activity, anti-obesity effects, vasodilator and antioxidant effects [1]. The main active ingredient of KP extract is the methoxyflovone derivative, which 5,7-dimethoxyflavone (DMF), 5,7,4'-trimethoxyflavone (TMF), and 3,5,7,3', 4'-pentamethoxyflavone (PMF) are commonly found. These compounds have low water solubility, high lipophilicity, and low bioavailability (about 1-4\%) [2-4]. Therefore, a potential transdermal delivery system should be developed to increase KP into the body and improve its therapeutic effects.

Organogel is a thermodynamically stable semisolid system, which an organic liquid phase is immobilized by a three-dimensional network of a gelator [5]. It has also been used as a potential transdermal drug delivery system. In recent years, organogel consisting of an aqueous solution of pluronic F127 TM (poloxamer 407) and lecithin have been reported to facilitate hydrophilic and lipophilic drugs through the skin [6,7].

The aim of the study was to develop the pluronic lecithin organogel formulations for enhancing transdermal delivery of KP extract. These formulations were prepared and their physicochemical properties, stability, and in vitro skin permeation were investigated.

\section{Materials and methods}

\subsection{Materials}

Isopropyl palmitate (IPM) was purchased from Nikkol, Singapore. Oleic acid and d-limonene were obtained from Sigma Aldrich, MO, USA. Lecithin (Emulmetik ${ }^{\circledR}$ 900) was purchased from Lucas Meyer Cosmetics, France. Poloxamer 407 (Kolliphor $^{\circledR}$ P 407) was obtained from BASF Corporation, Germany.

For KP extract, the dried rhizomes were purchased from Loei province, Thailand. The percolation technique with ethanol was employed to extract the methoxy flavones from KP.

\subsection{Preparation of KP extract-loaded pluronic lecithin organogel (PLO)}

PLO formulation containing oil phase $(5 \% \mathrm{w} / \mathrm{w}$ IPM, and $5 \% \mathrm{w} / \mathrm{w}$ oleic acid dissolved in $10 \% \mathrm{w} / \mathrm{w}$ lecithin) and aqueous phase $(20 \% \mathrm{w} / \mathrm{w}$ Poloxamer 407 dissolved in cold water) was prepared. For PLO-L formulation, $5 \% \mathrm{w} / \mathrm{w}$ d-limonene was added into $10 \% \mathrm{w} / \mathrm{w} \mathrm{KP}$ extract. Then, KP extract, oil phase, and the aqueous phase were mixed at ambient temperature.

\subsection{Physicochemical properties}

The $\mathrm{pH}$ value was evaluated using a $\mathrm{pH}$ meter (Metler Toledo, Seven compact S220) at room

\footnotetext{
Corresponding author: opanasopit $\mathrm{p} @$ su.suc.th
} 
temperature. For viscosity measurement, the samples were measured using a Brookfield viscometer (DV-III ultra, Brookfield Engineering Laboratories, USA) at 25 ${ }^{\circ} \mathrm{C}$. The stability of the formulations were monitored for 3 months under long-term (at room temperature) and accelerated $\left(40 \pm 2{ }^{\circ} \mathrm{C}, 75 \pm 5 \% \mathrm{RH}\right)$ conditions. The quantities of total methoxyflavones remaining in the formulations were determined at the beginning and after 3 months. Each experiment was determined in triplicate.

\subsection{In vitro skin permeation study}

Abdominal skin was obtained from naturally dead newborn pig from a farm in Ratchaburi province, Thailand. The subcutaneous layer was separated by medical scissors and surgical blades (thickness 0.6-0.7 $\mathrm{mm}$ ). Each skin was placed between donor and receiver compartment of Franz-type diffusion cells. Approximately $6 \mathrm{ml}$ of phosphate buffer saline (PBS, $\mathrm{pH}$ 7.4) was filled in the receiver part and stirred by a magnetic bar at $32{ }^{\circ} \mathrm{C}$. The $1 \mathrm{~g}$ of each formulation was added into the donor compartment. At 15, $30 \mathrm{~min}, 1,2$, 4,8 and $24 \mathrm{~h}, 0.8 \mathrm{~mL}$ of the receiver fluid was withdrawn, and fresh PBS was added to keep the constant volume of the receiver medium. The KP content was analyzed using a UPLC.

The cumulative amounts of total methoxyflavones permeated across the skin vs time were plotted, and the slope of each line represents a permeated flux. The permeability coefficients $\left(\mathrm{K}_{\mathrm{p}}\right)$ were calculated from the cumulative skin permeation profile using the flux, and the donor concentration $\left(C_{d}\right)$ of the formulations by equations (1).

$$
\mathrm{K}_{\mathrm{p}}=\text { Flux } / \mathrm{C}_{\mathrm{d}}
$$

\subsection{UPLC analysis}

Three methoxyflavones (DMF, TMF and PMF) were analyzed using an ACQUITY UPLC Core system (Waters Corporation, Milford, USA) binary solvent management and dual switching solvent/Degas/ ACQUITY TUV Detector and column heater. ACQUITY UPLC BEC C18 column $(2.1 \mathrm{~mm} \times 100 \mathrm{~mm}$, $1.7 \mu \mathrm{m}$, Waters ${ }^{\circledR}$ ) was used. The mobile phase consisted of acetonitrile and $0.5 \%$ formic acid in water $(15: 85$ to $85: 15)$, with the flow rate of $0.5 \mathrm{ml} / \mathrm{min}$ and $2.0 \mu \mathrm{L}$ injection volume. The operating temperature of column and samples was controlled at 38 and $25{ }^{\circ} \mathrm{C}$, respectively. The detection was operated using a UV detector at $335 \mathrm{~nm}$. The linearity range was $0.001-35$ $\mu \mathrm{g} / \mathrm{ml}$ and the $\mathrm{R}^{2}$ of the mean calibration plot was 0.9999 .

\subsection{Data analysis}

Each experiment was measured in triplicate. The results were reported as mean \pm standard deviation $(\mathrm{SD})$. The statistically significant differences between formulations were determined using analysis of variance (ANOVA) with an LSD post hoc test. The significance level was set at $\mathrm{p}<0.05$.

\section{Results and discussion}

\subsection{Physicochemical properties}

The amounts of DMF, PMF and TMF in KP extract were $10.39,9.18$, and $10.13 \%$, respectively. All formulations containing $10 \% \mathrm{w} / \mathrm{w}$ of $\mathrm{KP}$ extract were successfully prepared and exhibited brown opaque gel with $\mathrm{pH}$ 4.66. The percent total methoxyflavones content of PLO and PLO-L were $99.44 \pm 1.28 \%$ and $99.37 \pm$ $2.07 \%$, respectively. The viscosity of PLO and PLO-L were $42,256.96 \pm 771.47 \mathrm{cP}$ and $35,513.29 \pm 561.49 \mathrm{cP}$, respectively.

As shown in Fig. 1, the physicochemical properties of PLO-L formulation under storage conditions for 3 months exhibited a little change from the initial day of preparation, representing good formulation stability.
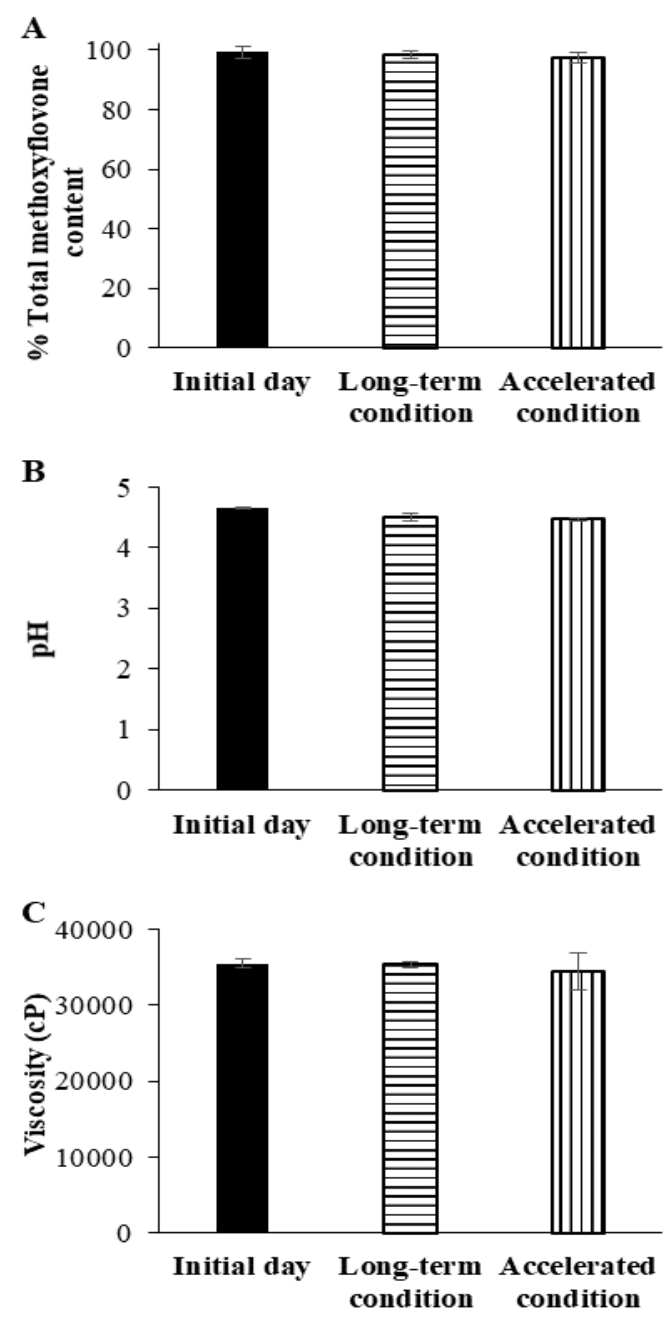

Fig. 1. Physicochemical properties (total methoxyflavone content (A), $\mathrm{pH}(\mathrm{B})$, and viscosity (C)) of PLO-L formulations at initial day and 3 months. Each value represents the mean \pm $\operatorname{SD}(n=3)$.

\subsection{In vitro skin permeation study}

As shown in Fig. 2, the permeation of KP extractloaded PLO-L and PLO formulation showed significantly higher total methoxyflavones permeated 
through the skin than KP extract in water, which the permeated flux and permeability coefficient were in the order: PLO-L > PLO > KP extract in water (Table 1). PLO-L provided the highest permeated flux of total methoxyflavones that was 2.00 -fold enhancement from KP extract. This result indicated that d-limonene play a role as skin permeation enhancer and intercalate into stratum corneum intercellular lipids, leading to increase lipid lamellae fluidity and enhance skin permeability of drug via an intercellular pathway [8].

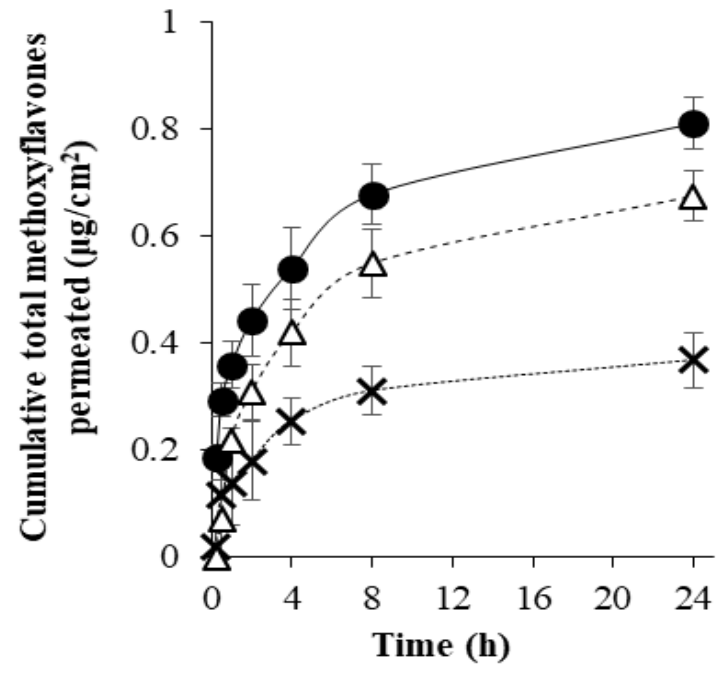

Fig. 2. Skin permeation profile of PLO $(\bullet)$ and PLO-L $(\Delta)$ compared with KP extract in water $(\mathrm{x})$. Each value represents the mean $\pm \mathrm{SD}(\mathrm{n}=3)$.

Table 1. The skin permeation flux and permeability coefficient $\left(\mathrm{K}_{\mathrm{p}}\right)$ of total methoxyflavones from KP extract in water, KP extract-loaded PLO, and KP extract-loaded PLO-L. Each value represents the mean $\pm \mathrm{SD}(\mathrm{n}=3) . *$ indicates significant different from KP extract in water.

\begin{tabular}{|c|c|c|}
\hline Formulations & Flux $\left(\mu \mathrm{g} \cdot \mathrm{cm}^{-2} \cdot \mathrm{h}^{-1}\right)$ & $\operatorname{Kp}\left(\mathrm{cm}^{-1} \mathrm{~h}^{-1}\right)\left(\mathrm{x} 10^{7}\right)$ \\
\hline $\begin{array}{l}\text { KP extract in } \\
\text { water }\end{array}$ & $0.013 \pm 0.001$ & $4.377 \pm 0.337$ \\
\hline PLO & $0.023 \pm 0.002^{*}$ & $7.744 \pm 0.673^{*}$ \\
\hline PLO-L & $0.026 \pm 0.002^{*}$ & $8.754 \pm 0.673^{*}$ \\
\hline
\end{tabular}

For PLO formulation that also enhanced total methoxyflavones permeability. Lecithin organogels might be efficient carriers for transdermal delivery, because lecithin promoted the skin permeation and partitioning of the drug into the skin layers. In addition, pluronic lecithin organogel is known as a new lecithin organogel. These systems have been developed for improving the skin permeation and stabilizing the formulation [9, 10]. Poloxamer 407 is a copolymer of polyoxyethylene and polyoxypropylene, which it can form a thermoreversible gel [11] and disrupts lipid layers of the skin stratum corneum [12]. Moreover, the PLO system has both oil and aqueous phases, so this system might be a potential transdermal delivery system of hydrophilic as well as lipophilic drugs $[6,7]$.

\section{Conclusion}

In this study, pluronic lecithin organogel consisting d-limonene could be a potential transdermal delivery system for KP extract. This system provided the highest skin permeability of total methoxylflavone and suitable physicochemical properties with good stability under long-term and accelerated condition.

The authors would like to thank Faculty of Pharmacy, Silpakorn University for financial supports, and Bangkok Lab \& Cosmetic Co., Ltd., for Kaempferia parviflora (KP) extract and standard methoxyflavones.

\section{References}

1. A. Chuthaputti, Journal Thai traditional \& Alternative Medicine 11, 1(2013)

2. K. Sutthanut, B. Sripanidkulchai, C. Yenjai, M. Jay. J. Chromatogr. A 1143, 1-2 (2007)

3. K. Sutthanut, X. Lu, B. Sripanidkulchai, C. Yenjai, M. Jay, J. Biomed. Nanotechnol 5, 2(2009).

4. C. Mekjaruskul, M. Jay, B. Sripanidkulchai, Drug. Metab. Dispos 40, 12(2012).

5. A. Vintiloiu, J. C. Leroux, J. Control. Release 125, 3 (2008).

6. B. L. Bramwell, L. V. A. Williams, Int. J. Pharm. Compd. 16, 1(2012).

7. H. Almeida, M. H. Amaral, P. Lobão, J. M. S. Lobo, J. Pharm. Pharm. Sci 15, 4(2012).

8. S. A. Ibrahim, S. K. Li, Int. J. Pharm 383, 12(2010).

9. R. Kumar, O. P. Katare, AAPS PharmSciTech 6, 2 (2005).

10. S. Raut, S. S. Bhadoriya, V. Uplanchiwar, V. Mishra, A. Gahane, S. K. Jain, Acta. Pharm. Sin. B 2, 1(2012).

11. G. Dumortier, J. L. Grossiord, F. Agnely, J. C. Chaumeil, Pharm. Res 23, 12 (2006).

12. V. Agrawal, V. Gupta, S. Ramteke, P. Trivedi, AAPS PharmSciTech 11, 4 (2010). 